\title{
End-to-End Performance Analysis of AF Relaying M2M Cooperative System
}

\author{
Lingwei $\mathrm{Xu}^{1}$, Hao Zhang ${ }^{1,2}$, Jingjing Wang ${ }^{3}$, Wei $\mathrm{Shi}^{3}$ and T. Aaron Gulliver ${ }^{2}$ \\ ${ }^{1}$ College of Information Science and Engineering, Ocean University of China, \\ Qingdao 266100, China \\ ${ }^{2}$ Department of Electrical and Computer Engineering, University of Victoria, \\ Victoria V8W 2Y2, Canada \\ ${ }^{3}$ College of Information Science \& Technology, Qingdao University of Science \& \\ Technology, Qingdao 266061, China \\ gaomilaojia2009@163.com; zhanghao@ouc.edu.cn; kathy1003@163.com; \\ agullive@ece.uvic.ca
}

\begin{abstract}
In this paper, the end-to-end performance of the mobile-relay-based mobile-to-mobile $(M 2 M)$ system with amplify-and-forward $(A F)$ relaying over $N$-Nakagami fading channels is investigated. The exact closed-form expressions for the lower bound on average symbol error probability (ASEP) and upper bound on channel capacity are derived. Then the ASEP and channel capacity performance under different conditions is evaluated to confirm the accuracy of the analysis. Results are presented which show that the fading coefficient, the number of cascaded components, the relative geometrical gain, and the power-allocation parameter have an important influence on the ASEP and channel capacity performance.
\end{abstract}

Keywords: M2M communication, N-Nakagami fading channels, amplify-and-forward, average symbol error probability, channel capacity

\section{Introduction}

Mobile-to-mobile (M2M) communication has attracted wide research interest in recent years. It is widely employed in many popular wireless communication systems, such as mobile ad-hoc networks and vehicle-to-vehicle communication system [1]. The classical Rayleigh, Rician, or Nakagami fading channels have been found not to be applicable in M2M communication. Experimental results and theoretical analysis demonstrate that cascaded fading distributions provide an accurate statistical model for M2M communications [2]. Cascaded Rayleigh fading channel, which involves the product of $N$ independent Rayleigh distributed random variables, is presented in [3]. For $N=2$, this reduces to double-Rayleigh fading model which has been found to be suitable in [4]. Afterwards, $N$-Nakagami channel is adopted to provide a realistic description of the M2M channel in [5]. For $N=2$, this reduces to double-Nakagami fading model in [6].

The demand for higher data rates with seamless service coverage and support for various applications in $\mathrm{M} 2 \mathrm{M}$ networks is greater than ever before. Cooperative diversity has been proposed as an ideal physical-layer solution for the high data-rate coverage required in M2M communication networks. Using fixed-gain amplify-and-forward (FAF) relaying scheme, [7] investigated pairwise error probability (PEP) for the cooperative inter-vehicular communication (IVC) system over double-Nakagami fading channels. In [8], the moment generating function (MGF) method was used to derive the exact symbol error rate (SER) and asymptotic SER expressions of the M2M system with decode-andforward (DF) relaying over double-Nakagami fading channels. Closed-form expressions 
for the outage probability of the multiple-mobile-relay-based M2M cooperative networks with relay selection and incremental AF relaying over $N$-Nakagami fading channels were derived in [9]. In [10], novel expressions for probability density function (PDF), and cumulative distribution function (CDF) of the end-to-end signal-to-noise ratio (SNR) were used to analyze the performance of two-way communication system with relay selection over $N$-Nakagami fading channels.

However, to the best knowledge of the author, the end-to-end ASEP and channel capacity performance of the mobile-relay-based AF relaying M2M system over $\mathrm{N}$ Nakagami fading channels has not been investigated in the literature. The absence of a closed form solution to the density function of the end-to-end signal to-noise ratio (SNR) in these relay systems makes the analysis cumbersome in most of these fading environments. This is more so with the case of $\mathrm{N}$-Nakagami fading channels where the SNR is treated as the product of several random variables. Moreover, most results mentioned above do not take the power allocation into account. This is an important issue and will be discussed in this paper as it affects the ASEP and channel capacity performance. In the present work, the main contributions are listed as follows:

First, we provide closed-form expressions for PDF and CDF of the complex $\mathrm{N}$ Nakagami channel coefficients. Then, we use those PDF expressions to derive exact closed-form expressions for the lower bound on ASEP and upper bound on channel capacity, respectively. Finally, based on the minimization of ASEP, we formulate a power-allocation problem to determine how the overall transmit power should be shared between the source and relay for performance optimization. In order to show the accuracy of the analytical results, the ASEP and channel capacity performance under different conditions is evaluated through numerical simulations. Results are presented which show that the fading coefficient, the number of cascaded components, the relative geometrical gain, and the power allocation parameter have a significant influence on the end-to-end ASEP and channel capacity performance.

The rest of the paper is organized as follows. The mobile-relay-based M2M system model is presented in Section 2. Section 3 provides the closed-form expressions for the end-to-end ASEP of PAM and PSK modulation, while in Section 4, the end-to-end channel capacity over $N$-Nakagami fading channels is examined. In Section 5 , the ASEP is optimized based on the power allocation parameter. Section 6 conducts Monte Carlo simulations to verify the analytical results. Concluding remarks are given in Section 7.

\section{The System Model}

Consider the simplest three-node cooperation model shown in Figure 1, namely mobile source (MS), mobile relay (MR), and mobile destination (MD) nodes. The nodes operate in half-duplex mode, which are equipped with a single pair of transmitter and receiver antennas.

According to [7], the relative gain of the MS to $\mathrm{MD}$ is $G_{\mathrm{SD}}=1$, the relative gains of the MS to MR and MR to MD links are $G_{\mathrm{SR}}=\left(d_{\mathrm{SD}} / d_{\mathrm{SR}}\right)^{v}$ and $G_{\mathrm{RD}}=\left(d_{\mathrm{SD}} / d_{\mathrm{RD}}\right)^{v}$, respectively, where $v$ is the path loss coefficient, $d_{\mathrm{SD}}, d_{\mathrm{SR}}$, and $d_{\mathrm{RD}}$ represent distances of MS to MD, MS to MR, and MR to MD links, respectively[11]. The relative geometrical gain $\mu=G_{\mathrm{SR}} / G_{\mathrm{RD}}$ indicates the location of MR with respect to MS and MD. When MR has the same distance to MS and MD, $\mu$ is $1(0 \mathrm{~dB})$. When MR is close to MD, $\mu$ has negative values. When MR is close to MS, $\mu$ has positive values. 


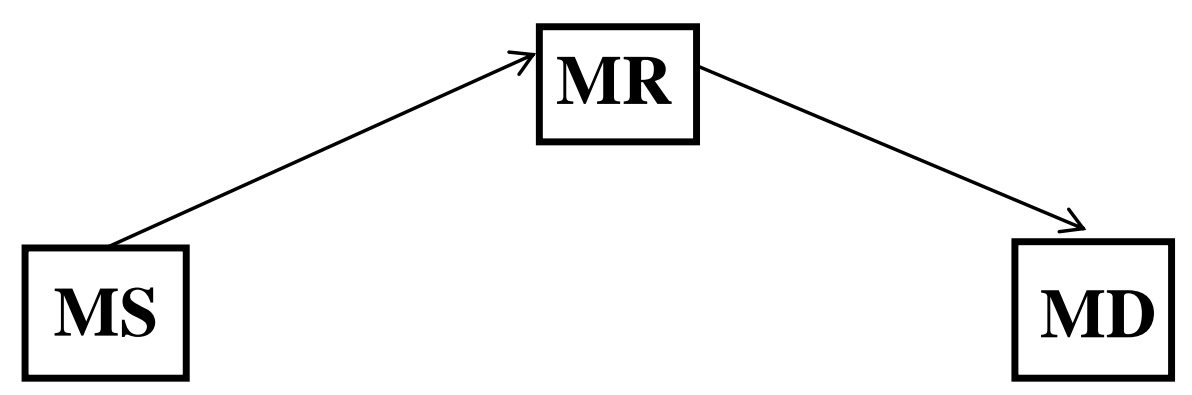

Figure 1. The System Model

Let $h=h_{k}, k \in\{\mathrm{SR}, \mathrm{RD}\}$, represent the complex channel coefficients of MS $\rightarrow \mathrm{MR}$, and $\mathrm{MR} \rightarrow$ MD links, respectively. $h$ follows $N$-Nakagami distribution, which is given as[5]

$$
h=\prod_{i=1}^{N} a_{i}
$$

Where $N$ is the number of cascaded components, $a_{i}$ is a Nakagami distributed random variable with $\mathrm{PDF}$ as

$$
f(a)=\frac{2 m^{m}}{\Omega^{m} \Gamma(m)} a^{2 m-1} \exp \left(-\frac{m}{\Omega} a^{2}\right)
$$

Where $\Gamma(\cdot)$ is the Gamma function, $m$ is the fading coefficient and $\Omega$ is a scaling factor. The PDF of $h$ is given by [5]

$$
f_{h}(h)=\frac{2}{h \prod_{i=1}^{N} \Gamma\left(m_{i}\right)} G_{0, N}^{N, 0}\left\lfloor\left. h^{2} \prod_{i=1}^{N} \frac{m_{i}}{\Omega_{i}}\right|_{m_{1}, \ldots, m_{N}} ^{-}\right\rfloor
$$

Where G[-] is the Meijer's G-function.

Let $y=\left|h_{k}\right|^{2}, k \in\{\mathrm{SR}, \mathrm{RD}\}$, namely, $y_{\mathrm{SR}}=\left|h_{\mathrm{SR}}\right|^{2}$, and $y_{\mathrm{RD}}=\left|h_{\mathrm{RD}}\right|^{2}$. The CDF of $y$ can be derived as [5]

$$
F_{y}(y)=\frac{1}{\prod_{i=1}^{N} \Gamma\left(m_{i}\right)} G_{1, N+1}^{N, 1}\left\lceil\left. y \prod_{i=1}^{N} \frac{m_{i}}{\Omega_{i}}\right|_{m_{1}, \ldots, m_{N}, 0} ^{1}\right\rfloor
$$

The corresponding PDF can be obtained as [5]

$$
f_{y}(y)=\frac{1}{y \prod_{i=1}^{N} \Gamma\left(m_{i}\right)} G_{0, N}^{N, 0}\left\lfloor\left. y \prod_{i=1}^{N} \frac{m_{i}}{\Omega_{i}}\right|_{m_{1}, \ldots, m_{N}} ^{-}\right\rceil
$$

During the first time slot, the received signal $r_{\mathrm{SR}}$ at the MR can be written as [7]

$$
r_{S R}=\sqrt{G_{S R} K E} h_{S R} x+n_{S R}
$$

Where $x$ denotes the transmitted signal, $n_{S R}$ is the zero-mean complex Gaussian random variables with variance $N_{0} / 2$ per dimension. During two time slots, the total energy used by MS and MR is $E$. $K$ is the power-allocation parameter which can control the fraction of power reserved for MS and MR.

During the second time slot, the received signal at the MD is therefore given by [7]

$$
r_{R D}=\sqrt{c E} h_{S R} h_{R D} x+n_{R D}
$$

Where $n_{\mathrm{RD}}$ is a conditionally zero-mean complex Gaussian random variable with variance $N_{0} / 2$ per dimension. 
For variable-gain $\mathrm{AF}(\mathrm{VAF}), c$ is given as [7]

$$
c=\frac{K(1-K) G_{S R} G_{R D} E / N_{0}}{1+K G_{S R}\left|h_{S R}\right|^{2} E / N_{0}+(1-K) G_{R D}\left|h_{R D}\right|^{2} E / N_{0}}
$$

The output SNR at the MD can then be calculated as [7]

$$
\gamma_{S R D}=\frac{\gamma_{S R} \gamma_{R D}}{1+\gamma_{S R}+\gamma_{R D}}
$$

Where

$$
\begin{gathered}
\gamma_{S R}=\frac{K G_{S R}\left|h_{S R}\right|^{2} E}{N_{0}}=K G_{S R}\left|h_{S R}\right|^{2} \bar{\gamma} \\
\gamma_{R D}=\frac{(1-K) G_{R D}\left|h_{R D}\right|^{2} E}{N_{0}}=(1-K) G_{R D}\left|h_{R D}\right|^{2} \bar{\gamma}
\end{gathered}
$$

As far as we know, a convenient mathematical method to obtain $\gamma_{\text {SRD }}$ exactly is still unachievable. Here, by using the same method in [8] and at high SNR by ignoring the 1 in (9), the SNR $\gamma_{\mathrm{SRD}}$ can be approximated as

$$
\gamma_{e q}=\frac{\gamma_{S R} \gamma_{R D}}{\gamma_{S R}+\gamma_{R D}}=\frac{1}{2} \frac{2}{\frac{1}{\gamma_{S R}}+\frac{1}{\gamma_{R D}}}
$$

By using the well-known inequality in [12], we can obtain an upper bound

$$
\gamma_{e q}<\gamma_{u p}=\min \left(\gamma_{S R}, \gamma_{R D}\right)
$$

When we use the upper bound $\gamma_{\mathrm{up}}$, instead of $\gamma_{\mathrm{SRD}}$, then the CDF is lower bounded, i.e.

$$
F_{\gamma_{S R D}}(r)>F_{\gamma_{u p}}(r)
$$

A new closed form expression for the tight lower bound on CDF of $\gamma_{\mathrm{SRD}}$ is given as [9]

$$
\begin{aligned}
& F_{\gamma_{u p}}(r)=\frac{1}{\prod_{t=1}^{N} \Gamma\left(m_{t}\right)} G_{1, N+1}^{N, 1}\left\lceil\left.\frac{r}{\overline{\gamma_{S R}}} \prod_{t=1}^{N} \frac{m_{t}}{\Omega_{t}}\right|_{m_{1}, \ldots, m_{N}, 0} ^{1}\right\rfloor \\
& +\frac{1}{\prod_{t t=1}^{N} \Gamma\left(m_{t t}\right)} G_{1, N+1}^{N, 1}\left\lceil\left.\frac{r}{\overline{\gamma_{R D}}} \prod_{t t=1}^{N} \frac{m_{t t}}{\Omega_{t t}}\right|_{m_{1}, \ldots, m_{N}, 0} ^{1}\right\rfloor- \\
& \frac{1}{\prod_{t=1}^{N} \Gamma\left(m_{t}\right) \prod_{t t=1}^{N} \Gamma\left(m_{t t}\right)} G_{1, N+1}^{N, 1}\left\lceil\left.\frac{r}{\overline{\gamma_{S R}}} \prod_{t=1}^{N} \frac{m_{t}}{\Omega_{t}}\right|_{m_{1}, \ldots, m_{N}, 0} ^{1}\right\rfloor \times \\
& G_{1, N+1}^{N, 1}\left\lceil\left.\frac{r}{\overline{\gamma_{R D}}} \prod_{t t=1}^{N} \frac{m_{t t}}{\Omega_{t t}}\right|_{m_{1}, \ldots, m_{N}, 0} ^{1}\right\rfloor
\end{aligned}
$$

By taking the first derivative of (15) with respect to $r$, the corresponding PDF can be obtained as 


$$
\begin{aligned}
& f_{\gamma_{u p}}(r)=\frac{1}{r \prod_{t=1}^{N} \Gamma\left(m_{t}\right)} G_{0, N}^{N, 0}\left\lceil\left.\frac{r}{=} \prod_{\gamma_{S R}}^{N} \frac{m_{t}}{\Omega_{t}}\right|_{m_{1}, \ldots, m_{N}} ^{-}\right\rfloor+ \\
& \frac{1}{r \prod_{t t=1}^{N} \Gamma\left(m_{t t}\right)} G_{0, N}^{N, 0}\left\lceil\left.\frac{r}{\overline{\gamma_{R D}}} \prod_{t t=1}^{N} \frac{m_{t t}}{\Omega_{t t}}\right|_{m_{1}, \ldots, m_{N}} ^{-}\right\rfloor-\frac{1}{r \prod_{t=1}^{N} \Gamma\left(m_{t}\right) \prod_{t t=1}^{N} \Gamma\left(m_{t t}\right)} \\
& \left\{G_{0, N}^{N, 0}\left\lceil\left.\frac{r}{=} \prod_{\gamma_{S R}}^{N} \frac{m_{t}}{\Omega_{t}}\right|_{m_{1}, \ldots, m_{N}} ^{-}\right\rceil G_{1, N+1}^{N, 1}\left\lceil\frac{r}{\overline{\gamma_{R D}}} \prod_{t t=1}^{N} \frac{m_{t t}}{\Omega_{t t}} \mid \begin{array}{l}
1 \\
m_{1}, \ldots, m_{N}, 0
\end{array}\right\rfloor\right.
\end{aligned}
$$

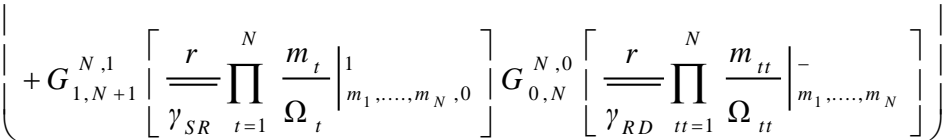

The lower bound on the OP of AF relaying is given as

$$
F_{\gamma_{S C B}}\left(r_{t h}\right)=F_{\gamma_{u p}}\left(r_{t h}\right)
$$

Where $r_{\text {th }}$ is a given threshold for correct detection at the MD.

\section{The ASEP of AF Relaying System}

The ASEP averaged over the fading channel is given by [13]

$$
P=\int_{0}^{\infty} P_{q}(r) f_{\gamma_{u p}}(r) d r
$$

Where $P_{q}(x)$ denotes the SEP of the $q$-ary modulation employed. We first consider $q$ ary PAM and then $q$-ary PSK.

\subsection{PAM}

The SEP of $q$-ary PAM modulation in an AWGN channel is given by [14]

$$
P_{q}(r)=2\left(1-\frac{1}{q}\right) Q\left(\sqrt{\frac{6 r}{q^{2}-1}}\right)
$$

Substituting (16) and (19) into (18), the ASEP of $q$-ary PAM over $N$-Nakagami fading channels is

$$
\begin{aligned}
& P_{P A M}=2\left(1-\frac{1}{q}\right) \int_{0}^{\infty} Q\left(\sqrt{\frac{6 r}{q^{2}-1}}\right) f_{\gamma_{u p}}(r) d r \\
& =\left(1-\frac{1}{q}\right)\left(G_{1}-G_{2}\right)
\end{aligned}
$$

With the help of [15, Eq. 21], $G_{1}$ can be given as

$$
\left.G_{1}=\frac{1}{\sqrt{\pi} \prod_{t=1}^{N} \Gamma\left(m_{t}\right)} G_{2, N+1}^{N, 2}\left\lfloor\frac{q^{2}-1}{3 \gamma_{S R}} \prod_{t=1}^{N} \frac{m_{t}}{\Omega_{t}}||_{m_{1}, \ldots, m_{N}, 0}^{1, \frac{1}{2}}\right\rfloor+\left.\frac{1}{\sqrt{\pi} \prod_{t t=1}^{N} \Gamma\left(m_{t t}\right)} G_{2, N+1}^{N, 2}\left|\frac{q^{2}-1}{3 \gamma_{R D}} \prod_{t t=1}^{N} \frac{m_{t t}}{\Omega_{t t}}\right|\right|_{m_{1}, \ldots, m_{N}, 0} ^{1, \frac{1}{2}}\right\rfloor
$$

$G_{2}$ can be given as 


$$
\begin{aligned}
& G_{2}=\frac{1}{\prod_{t=1}^{N} \Gamma\left(m_{t}\right) \prod_{t t=1}^{N} \Gamma\left(m_{t t}\right)} \int_{0}^{\infty} \operatorname{erfc}\left(\sqrt{\frac{3 r}{q^{2}-1}}\right) \frac{1}{r} \times \\
& \left\{\begin{array}{l}
G_{0, N}^{N, 0}\left\lceil\left.\frac{r}{=} \prod_{\gamma_{S R}}^{N} \frac{m_{t}}{\Omega_{t}}\right|_{m_{1}, \ldots, m_{N}}\right\rfloor G_{1, N+1}^{N, 1}\left\lceil\left.\frac{r}{\overline{\gamma_{R D}}} \prod_{t t=1}^{N} \frac{m_{t t}}{\Omega_{t t}}\right|_{m_{1}, \ldots, m_{N}, 0} ^{1}\right\rfloor \mid \\
\left.+G_{1, N+1}^{N, 1}\left\lceil\left.\frac{r}{\overline{\gamma_{S R}}} \prod_{t=1}^{N} \frac{m_{t}}{\Omega_{t}}\right|_{m_{1}, \ldots, m_{N}, 0} ^{1}\right\rfloor G_{0, N}^{N, 0}\left|\frac{r}{\overline{\gamma_{R D}}} \prod_{t t=1}^{N} \frac{m_{t t}}{\Omega_{t t}}\right|_{m_{1}, \ldots, m_{N}}\right\rfloor \mid
\end{array}\right. \\
& d r \\
& =\frac{1}{\sqrt{\pi} \prod_{i=1}^{N} \Gamma\left(m_{i}\right) \prod_{t t=1}^{N} \Gamma\left(m_{t t}\right)}\left(\begin{array}{c|c}
G_{2,1: 0, N: 1, N+1}^{0,2: N, 0: N, 1} & 1, \frac{1}{2} \mid \\
0 & -
\end{array} \mid\right. \\
& \left.m_{1}, \ldots, m_{N}, 0 \mid \frac{q^{2}-1}{3 \overline{\gamma_{S R}}} \prod_{t=1}^{N} \frac{m_{t}}{\Omega_{t}}, \frac{q^{2}-1}{3 \overline{\gamma_{R D}}} \prod_{t t=1}^{N} \frac{m_{t t}}{\Omega_{t t}}\right\rceil+G_{2,1: 0, N: 1, N+1}^{0,2: N, 0: N, 1}\left\lceil\begin{array}{c}
1, \frac{1}{2} \\
0
\end{array} \mid\right. \\
& \left.\left.m_{1}, \ldots, m_{N}\left|m_{1}, \ldots, m_{N}, 0\right| \frac{q^{2}-1}{3 \overline{\gamma_{R D}}} \prod_{t t=1}^{N} \frac{m_{t t}}{\Omega_{t t}}, \frac{q^{2}-1}{3 \gamma_{S R}} \prod_{t=1}^{N} \frac{m_{t}}{\Omega_{t}}\right\rfloor \mid\right)
\end{aligned}
$$

\subsection{PSK}

The SEP of $q$-ary PSK modulation in an AWGN channel is given by [14]

$$
P_{q}(r)=2 Q\left(\sqrt{2 \sin ^{2} \frac{\pi}{q} r}\right)-\frac{1}{\pi} \int_{\frac{\pi}{2}-\frac{\pi}{q}}^{\frac{\pi}{2}} e^{-r \frac{\sin ^{2} \pi / q}{\cos ^{2} \theta}} d \theta
$$

For large SNR and large values of $q$, the SEP of $q$-ary PSK in an AWGN channel can be approximated as

$$
P_{q}(r) \approx 2 Q\left(\sqrt{2 \sin ^{2} \frac{\pi}{q} r}\right)
$$

Substituting (16) and (24) into (18), the ASEP of $q$-ary PSK over $N$-Nakagami fading channels can be approximated as

Where

$$
\begin{aligned}
& P_{P S K}(r) \approx \int_{0}^{\infty} 2 Q\left(\sqrt{2 \sin ^{2} \frac{\pi}{q} r}\right) f_{\gamma_{u p}}(r) d r \\
& =G_{3}-G_{4}
\end{aligned}
$$

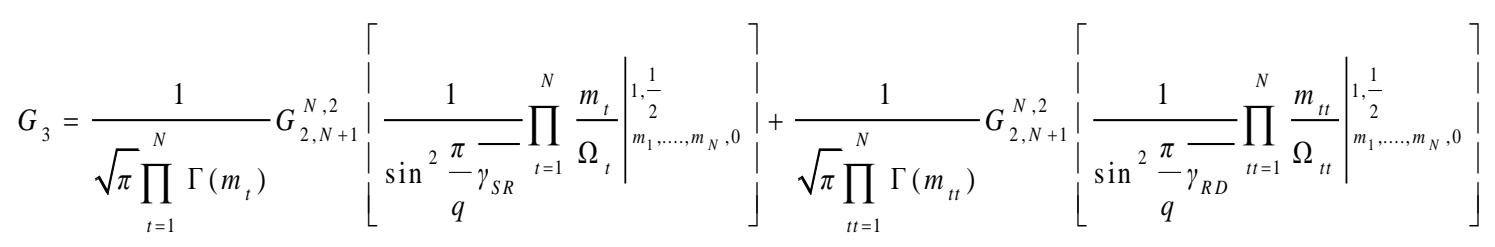




$$
\begin{aligned}
& G_{4}=\frac{1}{\sqrt{\pi} \prod_{t=1}^{N} \Gamma\left(m_{t}\right) \prod_{t t=1}^{N} \Gamma\left(m_{t t}\right)} \\
& \left\{\begin{array}{c|c|c}
G_{2,1: 0, N: 1, N+1}^{0,2: N, 0: N, 1}\left|\begin{array}{c}
1, \frac{1}{2} \\
2
\end{array}\right| m_{1}, \ldots, m_{N} & - \\
0 & \mid m_{1}, \ldots, m_{N}, 0 & \frac{1}{\sin ^{2} \frac{\pi}{q} \gamma_{S R}} \prod_{t=1}^{N} \frac{m_{t}}{\Omega_{t}}, \frac{1}{\sin ^{2} \frac{\pi}{q} \gamma_{R D}} \prod_{t t=1}^{N} \frac{m_{t t}}{\Omega_{t t}} \mid
\end{array}\right\rfloor
\end{aligned}
$$

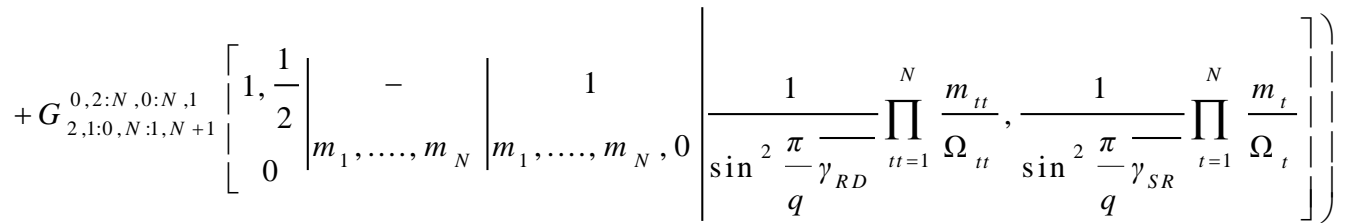

\section{The Capacity of AF Relaying System}

The capacity for AF relaying over $N$-Nakagami fading channels is given as [14]

$$
\begin{aligned}
& C=\log _{2}(e) \int_{0}^{\infty} \ln (1+r) f_{\gamma_{u p}}(r) d r \\
& =\log _{2}(e)\left(U_{1}-U_{2}\right)
\end{aligned}
$$

Where

$$
\begin{aligned}
& U_{1}=\frac{1}{\prod_{t=1}^{N} \Gamma\left(m_{t}\right)} G_{2, N+2}^{N+2,1}\left\lfloor\left.\frac{1}{\left\lfloor\gamma_{S R}\right.} \prod_{t=1}^{N} \frac{m_{t}}{\Omega_{t}}\right|_{m_{1}, \ldots, m_{N}, 0,0} ^{0,1}\right\rfloor+\frac{1}{\prod_{t=1}^{N} \Gamma\left(m_{t t}\right)} G_{2, N+2}^{N+2,\lceil}\left\lfloor\frac{1}{\left\lfloor\gamma_{R D}\right.} \prod_{t t=1}^{N} \frac{m_{t t}}{\Omega_{t t}} \mid \begin{array}{l}
0,1 \\
m_{1}, \ldots, m_{N}, 0,0
\end{array}\right\rfloor \\
& U_{2}=\frac{1}{\prod_{t=1}^{N} \Gamma\left(m_{t}\right) \prod_{t t=1}^{N} \Gamma\left(m_{t t}\right)} \times
\end{aligned}
$$

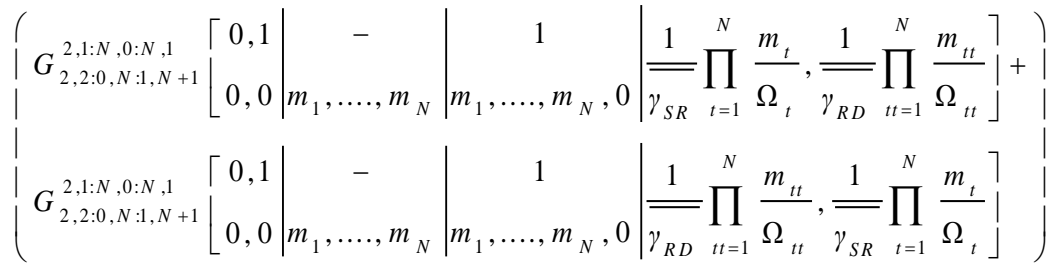

\section{Optimized Power Allocation}

To optimize the power allocation, the resulting ASEP needs to be minimized with respect to the power-allocation parameter $K(0 \leq K \leq 1)$. 


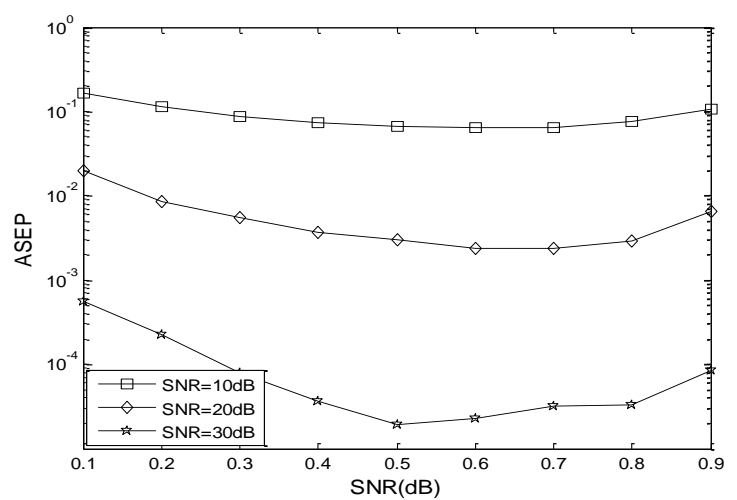

\section{Figure 2. The Effect of the Power-Allocation Parameter $K$ on the ASEP Performance}

Figure 2 presents the effect of the power allocation parameter $K$ on the ASEP performance of AF relaying M2M cooperative system over $N$-Nakagami fading channels. The number of cascaded components is $N=2$, the fading coefficient is $m=2$, and the relative geometrical gain is $\mu=-5 \mathrm{~dB}$. These results show that the ASEP performance is improved with an increase in the SNR. For example, with $K=0.7$, the ASEP is $6.5 \times 10^{-2}$ for $\mathrm{SNR}=10 \mathrm{~dB}, 2.4 \times 10^{-3}$ for $\mathrm{SNR}=20 \mathrm{~dB}$, and $3.2 \times 10^{-5}$ for $\mathrm{SNR}=30 \mathrm{~dB}$. When $\mathrm{SNR}=10 \mathrm{~dB}$, the optimum value of $K$ is 0.62 , when $\mathrm{SNR}=20 \mathrm{~dB}$, the optimum value of $K$ is 0.70 , and when $\mathrm{SNR}=30 \mathrm{~dB}$, the optimum value of $K$ is 0.56 . This indicates that equal power allocation (EPA) is not always the best approach.

From Figure 2, it can readily be checked that these expressions are convex functions with respect to $K$. The convexity of the ASEP functions under consideration guarantees that the local minimum found through optimization will indeed be a global minimum. Unfortunately, an analytical solution for power allocation values in the general case is very difficult. We resort to numerical methods to solve this optimization problem. The optimum power allocation (OPA) values can be obtained a priori for given values of operating SNR and propagation parameters. The OPA values can be stored for use as a lookup table in practical implementation.

In Table 1, we present optimum values of $K$ for $N$-Nakagami fading channels with BPSK modulation. We assume that the number of cascaded components is $N=2$, the fading coefficient is $m=2$, and the relative geometrical gain is $\mu=5,0,-5 \mathrm{~dB}$. We observe from Table 1 that when MR is close to MS, less than 50\% of power is required by MS to achieve optimum performance; when MR is equidistant from MS and MD, nearly $50 \%$ of power is required by MS to achieve optimum performance; when MR is close to MD, more than $50 \%$ of power is required by MS to achieve optimum performance.

Figure 3 presents the effect of the EPA and OPA on the ASEP performance with BPSK modulation. For OPA, the values of $K$ are used in Table 1 for $\mu=-5 \mathrm{~dB}$. For EPA, $K=0.5$. These results show that the ASEP performance of OPA is better than that of EPA. For example, with $\mathrm{SNR}=25 \mathrm{~dB}$, the ASEP is $2.2 \times 10^{-4}$ for OPA, while $4 \times 10^{-4}$ for EPA.

Table 1. OPA Parameters $K$

\begin{tabular}{|c|c|c|c|}
\hline SNR & $\mu=5 \mathrm{~dB}$ & $\mu=0 \mathrm{~dB}$ & $\mu=-5 \mathrm{~dB}$ \\
\hline 5 & 0.46 & 0.47 & 0.56 \\
\hline 10 & 0.43 & 0.52 & 0.62 \\
\hline 15 & 0.40 & 0.49 & 0.64 \\
\hline 20 & 0.39 & 0.52 & 0.70 \\
\hline 25 & 0.21 & 0.54 & 0.72 \\
\hline 30 & 0.26 & 0.41 & 0.56 \\
\hline
\end{tabular}




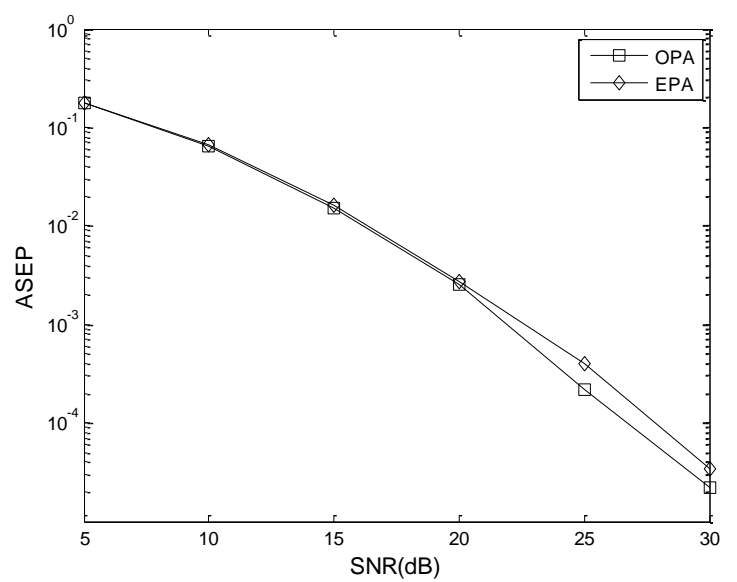

Figure 3. The Effect of the EPA and OPA on the ASEP Performance

\section{Numerical Results}

In this section, we present Monte-Carlo simulations and numerical methods to confirm the derived analytical results. Additionally, random number simulation was done to confirm the validity of the analytical approach. All the computations were done in MATLAB and some of the integrals were verified through MAPLE. The links between MS to MR and MR to MD are modeled as $N$-Nakagami distribution. The total energy is $E$ $=1$. The fading coefficient is $m=1,2,3$, the number of cascaded components is $N=2,3,4$, and the relative geometrical gain is $\mu=5 \mathrm{~dB}, 0 \mathrm{~dB},-5 \mathrm{~dB}$, respectively. In Figure 5, 6, 7, 8, we only want to present the effect of $m, N, \mu$ on the ASEP and channel capacity, respectively, so the value of $K$ should remain unchanged. Here, we choose $K=0.5$.

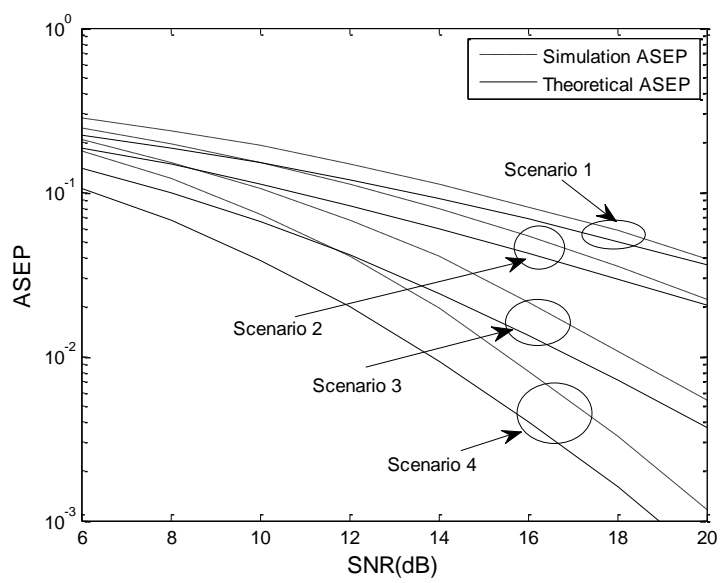

Figure 4. The ASEP Performance Over $\mathrm{N}$-Nakagami Fading Channels

Figure 4 presents the ASEP performance of AF relaying M2M system over $N$ Nakagami fading channels. The relative geometrical gain is $\mu=0 \mathrm{~dB}$. The power-allocation parameter is $K=0.5$. Here, we consider the following scenarios based on the combinations of the number of cascaded components $N$ and fading coefficient $m$ :

(1) Scenario 1: $m_{\mathrm{SR}}=1, m_{\mathrm{RD}}=1$ and $N_{\mathrm{SR}}=N_{\mathrm{RD}}=2$.

(2)Scenario 2: $m_{\mathrm{SR}}=2, m_{\mathrm{RD}}=1$ and $N_{\mathrm{SR}}=N_{\mathrm{RD}}=2$.

(3)Scenario 3: $m_{\mathrm{SR}}=2, m_{\mathrm{RD}}=2$ and $N_{\mathrm{SR}}=N_{\mathrm{RD}}=2$.

(4)Scenario 4: $m_{\mathrm{SR}}=3, m_{\mathrm{RD}}=3$ and $N_{\mathrm{SR}}=N_{\mathrm{RD}}=2$.

From Figure 4, we can obtain that, the numerical simulation results coincide with the 
theoretical results well, and the accuracy of the analytical results is verified. The difference between the simulation results and theoretical results decreases as the SNR increases. For example, in Scenario 3, when $S N R=10 \mathrm{~dB}$, the simulation ASEP is $1 \times 10^{-1}$, the theoretical ASEP is $6.6 \times 10^{-2}$, while SNR $=14 \mathrm{~dB}$, the simulation ASEP is $4.1 \times 10^{-2}$, the theoretical ASEP is $2.4 \times 10^{-2}$.

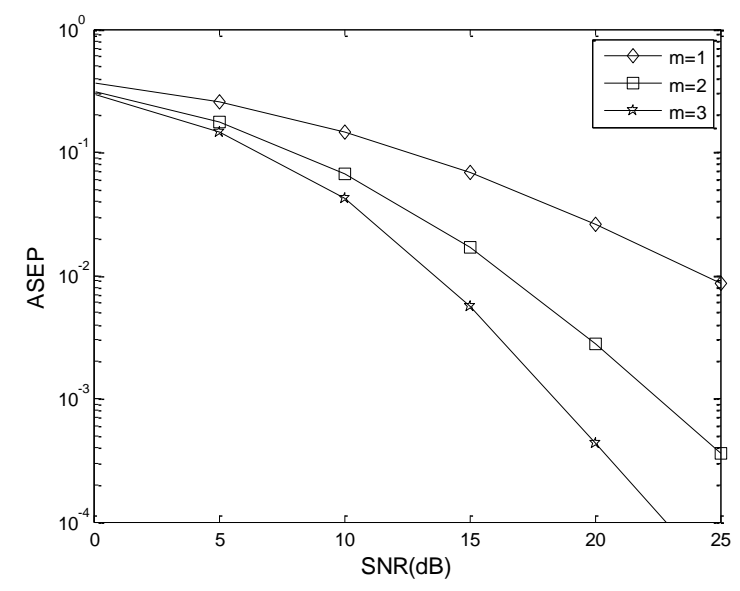

Figure 5. The Effect of the Fading Coefficient $\boldsymbol{m}$ on the ASEP Performance

Figure 5 presents the effect of the fading coefficient $m$ on the ASEP performance. The number of cascaded components is $N=2$.The fading coefficient is $m=1,2,3$. The relative geometrical gain is $\mu=-5 \mathrm{~dB}$. The power-allocation parameter is $K=0.5$. With the fading coefficient $m$ increased, the ASEP performance is improved. For example, when SNR $=15$ $\mathrm{dB}, m=1$, the ASEP is $6.8 \times 10^{-2}, m=2$, the ASEP is $1.7 \times 10^{-2}, m=3$, the ASEP is $5.6 \times 10^{-3}$. When the $m$ is fixed, with the increase of SNR, the ASEP is reduced gradually.

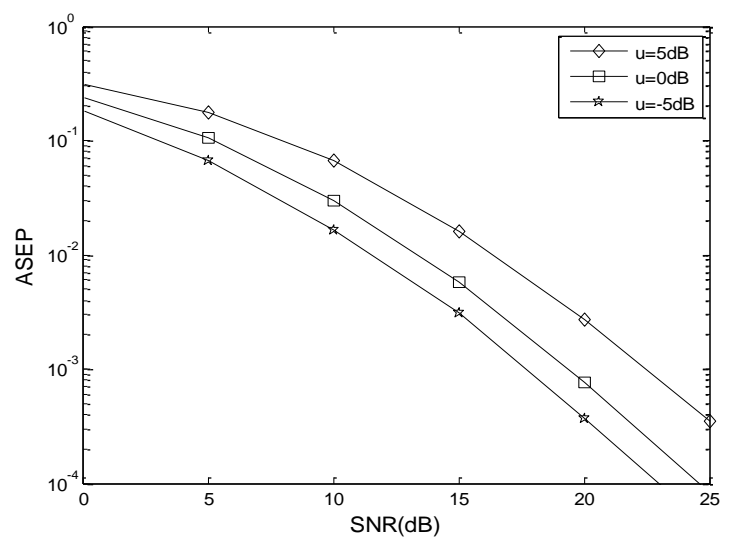

\section{Figure 6. The Effect of the Relative Geometrical Gain $\mu$ on the ASEP Performance}

Figure 6 presents the effect of the relative geometrical gain $\mu$ on the ASEP performance. The number of cascaded components is $N=2$. The fading coefficient is $m=2$. The relative geometrical gain is $\mu=5 \mathrm{~dB}, 0 \mathrm{~dB},-5 \mathrm{~dB}$. The power-allocation parameter is $K=0.5$. The ASEP performance is improved as $\mu$ reduced. For example, when $\mathrm{SNR}=15 \mathrm{~dB}$, $\mu=5 \mathrm{~dB}$, the ASEP is $2 \times 10^{-2}, \mu=0 \mathrm{~dB}$, the ASEP is $5 \times 10^{-3}, \mu=-5 \mathrm{~dB}$, the ASEP is $3 \times 10^{-3}$. This indicates that the best location for the relay is near the destination. For a fixed $\mu$, an increase in the SNR reduces the ASEP. 


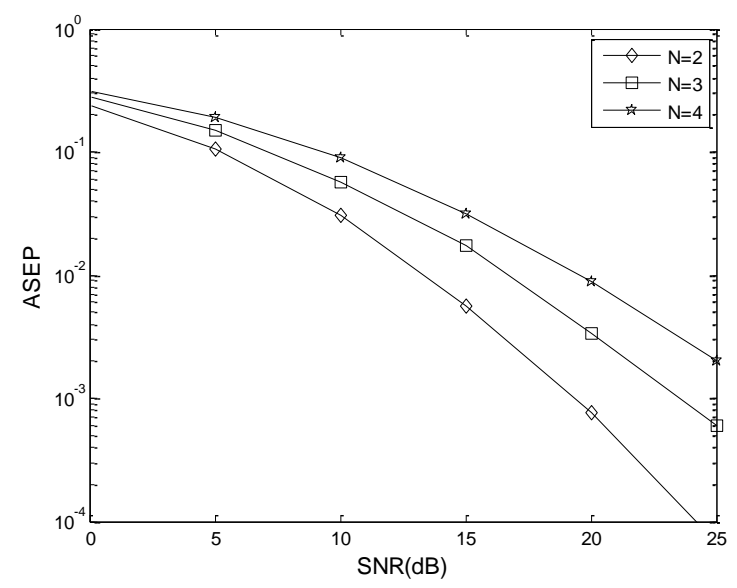

\section{Figure 7. The Effect of the Number of Cascaded Components $\mathbf{N}$ on the ASEP Performance}

Figure 7 presents the effect of the number of cascaded components $N$ on the ASEP performance. The number of cascaded components is $N=2,3$, 4, which respectively denotes the 2-Nakagami, 3-Nakagami, 4-Nakagami fading channels. The fading coefficient is $m=2$. The relative geometrical gain is $\mu=0 \mathrm{~dB}$. The power-allocation parameter is $K=0.5$. Simulation results show that the ASEP performance is degraded as $N$ increased. For example, when $\mathrm{SNR}=10 \mathrm{~dB}$, the ASEP is $3 \times 10^{-2}$ with $N=2,5.7 \times 10^{-2}$ with $N=3,8.9 \times 10^{-2}$ with $N=4$. This is because the fading severity of the cascaded channels increases as $N$ increased. For fixed $N$, an increase in the SNR reduces the ASEP, as expected.

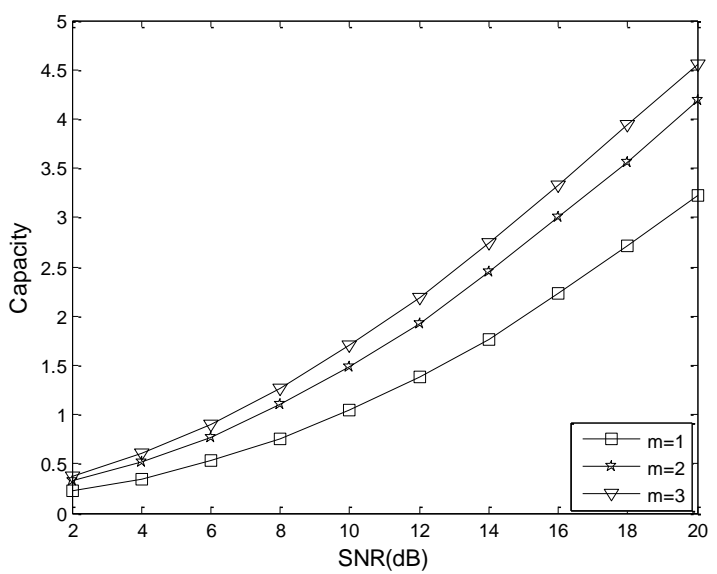

\section{Figure 8. The Channel Capacity Performance over N-Nakagami Fading Channels}

Figure 8 presents the channel capacity performance of AF relaying M2M system over $N$-Nakagami fading channels. The relative geometrical gain is $\mu=0 \mathrm{~dB}$. The powerallocation parameter is $K=0.5$. The number of cascaded components is $N=2$. The fading coefficient is $m=1,2,3$. Simulation results show that the channel capacity is improved as the fading coefficient $m$ is increased. For example, when $\mathrm{SNR}=14 \mathrm{~dB}$, the channel capacity is $1.76 \mathrm{bps} / \mathrm{Hz}$ for $m=1,2.45 \mathrm{bps} / \mathrm{Hz}$ for $m=2$, and $2.74 \mathrm{bps} / \mathrm{Hz}$ for $m=3$. This is because the fading severity of an $N$-Nakagami channel is less for a larger $m$. For fixed $m$, an increase in the SNR again increases the channel capacity. 


\section{Conclusions}

The exact closed-form expressions for the end-to-end ASEP and channel capacity of the AF relaying M2M system over $N$-Nakagami fading channels are derived in this paper. The simulation results show that the fading coefficient $m$, the number of cascaded components $N$, the relative geometrical gain $\mu$, and the power-allocation parameter $K$ have an important influence on the ASEP and channel capacity performance. The derived ASEP and channel capacity expressions can be used to evaluate the performance of the vehicular communication systems such as inter-vehicular communications, intelligent highway applications and mobile ad-hoc applications. In the future, we will consider the impact of the correlated channels on the ASEP and channel capacity performance of the AF relaying M2M system.

\section{Acknowledgments}

The authors would like to thank the referees and editors for providing very helpful comments and suggestions. This project was supported by National Natural Science Foundation of China (No. 61304222, No. 61301139), Natural Science Foundation of Shandong Province (No.ZR2012FQ021), Shandong Province Outstanding Young Scientist Award Fund (No. 2014BSE28032), Fundamental Research Funds for the Central Universities (No. 14CX02139A).

\section{References}

[1] S. Mumtaz, K. M. S. Huq and J. Rodriguez, "Direct mobile-to-mobile communication: Paradigm for 5G", IEEE Wireless Communications, vol. 21, no. 5, (2014), pp. 14-23.

[2] H. Shin and M. Win, "MIMO diversity in the presence of double scattering", IEEE Transactions on Vehicular Technology, vol. 54, no. 7, (2008), pp. 2976-2996.

[3] J. Salo, H. E. Sallabi and P. Vainikainen, "The distribution of the product of independent Rayleigh random variables", IEEE Transactions on Antennas and Propagation, vol. 54, no. 2, (2006), pp. 639-643.

[4] M. Uysal, "Diversity Analysis of Space-time Coding in Cascaded Rayleigh Fading Channels", IEEE Communication Letters, vol. 10, no.3, (2006), pp. 165-167.

[5] G. K. Karagiannidis, N. C. Sagias and P. T. Mathiopoulos, "N*Nakagami: a novel stochastic model for cascaded fading channels", IEEE Transactions on Communications, vol. 55, no. 8, (2007), pp. 14531458.

[6] F. K.Gong, J. Ge and N. Zhang, "SER Analysis of the Mobile-Relay-Based M2M Communication over Double Nakagami-m Fading Channels", IEEE Communication Letters, vol. 15, no.1, (2011), pp. 34-36.

[7] H. Ilhan, M. Uysal and I. Altunbas, "Cooperative Diversity for Intervehicular Communication: Performance Analysis and Optimization," IEEE Transactions on Vehicular Technology, vol. 58, no.7, (2009), pp. 3301-3310.

[8] F. K. Gong, P. Ye, Y. Wang and N. Zhang, "Cooperative mobile-to-mobile communications over double Nakagami-m fading channels", IET Communications, vol. 6, no. 18, (2012), pp. 3165-3175.

[9] L. W. Xu, H. Zhang, T. T. Lu and T. A. Gulliver, "Performance Analysis of the IAF Relaying M2M Cooperative Networks over N-Nakagami Fading Channels", Journal of Communications, vol. 10, no.3, (2015), pp. 185-191.

[10] H. Ilhan, "Relay Selection in Two-Way Cooperative Systems", Wireless Personal Communications, vol. 77, no.2, (2014), pp. 1329-1341.

[11] H. Ochiai, P. Mitran and V. Tarokh, "Variable-rate two-phase collaborative communication protocols for wireless networks", IEEE Transactions on Vehicular Technology, vol. 52, no. 9, (2006), pp. 4299-4313.

[12] P. A. Anghel and M. Kaveh, "Exact symbol error probability of a cooperative network in a Rayleighfading environment", IEEE Transactions on Wireless Communications, vol. 3, no. 5, (2004), pp. 14161421.

[13] J. G. Proakis, "Digital Communications", McGraw-Hill, New York, NY, USA, 4th edition, (2001).

[14] M. K. Simon and M. S. Alouini, "Digital Communication over Fading Channels", Wiley, New York, 2nd edition, (2004).

[15] V. S. Adamchik and O. I .Marichev, "The algorithm for calculating integrals of hypergeometric type functions and its realization in reduce system", in Proc. of Int. Symposium on Symbolic and Algebraic Computation, Tokyo, Japan, (1990), pp. 212-224. 


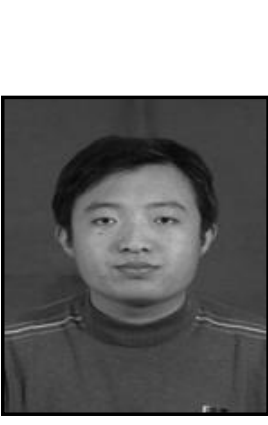

\section{Authors}

Lingwei Xu, received his B.E. degree in Communication Engineering from Qingdao Technological University, China in 2011. He received his M.E. degree in Electronics and Communication Engineering from Ocean University of China, China in 2013. Now he is a Ph.D. candidate in Ocean University of China. His research interests include ultra-wideband radio systems, MIMO wireless systems, and M2M wireless communications.

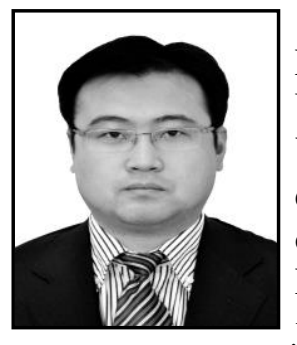

Hao Zhang, received his B.E. degree in Telecommunications Engineering and Industrial Management from Shanghai Jiaotong University, China in 1994. He received his MBA degree from New York Institute of Technology, USA in 2001. He received his Ph.D. degree in Electrical and Computer Engineering from the University of Victoria, Canada in 2004. From 1994 to 1997, he was the Assistant President of ICO Global Communication Company. In 2000, he joined Microsoft Canada as a Software Engineer, and was Chief Engineer at Dream Access Information Technology, Canada from 2001 to 2002. He is currently a professor in the Department of Electronic Engineering at Ocean University of China and an adjunct professor in the Department of Electrical and Computer Engineering at the University of Victoria. His research interests include ultrawideband radio systems, MIMO wireless systems, cooperative communication networks and spectrum communications.

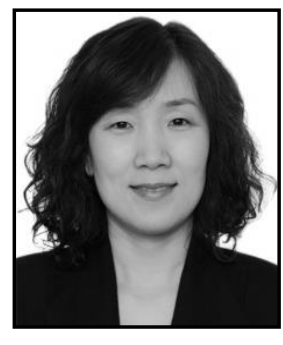

Jingjing Wang was born in Anhui, China, in 1975. She received her B.S. degree in Industrial Automation from Shandong University, Jinan, China, in 1997, the M.S. degree in Control Theory and Control Engineering, Qingdao University of Science \& Technology, Qingdao, China, in 2002, and the Ph.D. degree in Computer Application Technology, Ocean University of China, Qingdao, China, in 2012. From 2002 to now, she is an associate professor at the College of Information Science \& Technology, Qingdao University of Science \& Technology. Her research interests include $60 \mathrm{GHz}$ wireless communication, $60 \mathrm{GHz}$ wireless position technology, ultra wideband radio systems, and cooperative communication networks.

T. Aaron Gulliver, received his Ph.D. degree in Electrical and Computer Engineering from the University of Victoria, Victoria, BC, Canada in 1989. He is a professor in the Department of Electrical and Computer Engineering. From 1989 to 1991 he was employed as a Defense Scientist at Defense Research Establishment Ottawa, Ottawa, ON, Canada. He has held academic positions at Carleton University, Ottawa, and the University of Canterbury, Christchurch, New Zealand. He joined the University of Victoria in 1999 and is a Professor in the Department of Electrical and Computer Engineering. In 2002 he became a Fellow of the Engineering Institute of Canada, and in 2012 a Fellow of the Canadian Academy of Engineering. He is also a senior member of IEEE. His research interests include information theory and communication theory, and ultra wideband communication. 
International Journal of Multimedia and Ubiquitous Engineering Vol.10, No.9 (2015) 